\title{
Giant Cells Osseous Tumor in the Tarsal Canal after Lateral Ankle Sprain
}

\author{
Marcello Lughi ${ }^{1}$ \\ ${ }^{1}$ AUSL della Romagna, Presidio Ospedaliero di Forlì, Forlì, Italy \\ Joints 2018;6:65-67.
}

Address for correspondence Marcello Lughi, MD, Via Bellaria 7, 47521 Cesena (FC), Forlì, Italy (e-mail: mlughi@alice.it).
Abstract
Keywords
- ankle
- sprain
- subtalar joint
- giant cells osseous tumor

\begin{abstract}
Ankle sprain can cause injuries to the anatomic structures surrounding the tibiotarsal joint. A possible extra-articular pathology is to be hypothesized and diagnosed as early as possible. The subtalar joint, for anatomical and functional reasons, is one of the most damaged joints following an ankle sprain. In spite of this, its involvement is often underestimated. The clinical case presented in the present article is referred to a giant cells osseous tumor in the tarsal canal that was diagnosed 2 months after an inversion ankle sprain.
\end{abstract}

\section{Introduction}

Ankle sprain can cause lesions to intra-articular and extraarticular structures. ${ }^{1-4}$ Articular lesions are mainly represented by capsular and ligamentous injuries (predominantly on the lateral side) and osteochondral lesions. In addition to these lesions, that we can define as acute, chronic lesions can more often be caused by soft tissue and bony impingement at the anterior and posterior compartment of the ankle. In addition, we can also find extra-articular lesions that may affect various anatomic structures around the ankle, such as tendons (peroneals tendon, posterior tibial tendon) and capsuloligament structures (subtalar joint and the spring ligament complex). Furthermore, talus fractures (i.e., fractures of the posteromedial and or posterolateral tuberosity, and fractures of the lateral portion) and calcaneus fractures can occur.

From the anatomical, biomechanical, and functional point of view, subtalar joint is in a close correlation with the ankle. Very often, the subtalar space is affected by trauma at the same time as the ankle joint. Subtalar joint is a district that contributes to the stability and function of the hindfoot and it has a very complex ligamentous apparatus composed of many ligaments.

Of the ligamentous apparatus of the subtalar joint, the most known is its lateral portion, which comprises a series of separate ligaments in three layers: superficial (lateral root of the peroneal retinaculum and lateral calcaneus ligament), intermediate (the intermediate root of the peroneal retinaculum and the cervical ligament), and deep (the medial root

received

May 18, 2017

accepted after revision

November 21, 2017

published online

January 2, 2018 of the peroneal retinaculum and the interosseous talocalcaneal ligament).

In the lateral aspect of the subtalar joint, also a ligament that goes from the apex of the fibula to the calcaneus can be seen. ${ }^{5-10}$ Medially, we find the spring ligament complex. ${ }^{11-16}$

Subtalar joint, especially in its component of the canal and sinus tarsi, is richly innervated and vascularized. Vascular and nerve endings can deepen in the tarsal canal and are particularly represented in the deep adipose tissue. The most common traumatic mechanism at the base of ankle sprain is in inversion (tibiotarsal plantar flexion plus hindfoot supination plus internal rotation of the mid-forefoot).

In a supination trauma, the ligaments often affected are those of the lateral compartment of the ankle, but it is also possible to stress the lateral ligaments of the subtalar joint.

Several studies reported multiple lesions associated with ankle sprain, both acute and chronic. Despite this evidence and the ever-increasing knowledge of these lesions, in rare cases some lesions are misunderstood as results of insufficient clinical and instrumental diagnostic approach. ${ }^{17-19}$ The clinical case presented herein is unique and original for etiology, anatomical features, and localization.

\section{Case Description}

This study presents a 26-year-old patient case who 9 months before clinical observation had suffered an ankle supination sprain playing soccer. After a first treatment for 1 week with
DOI https://doi.org/

10.1055/s-0037-1617416. ISSN 2282-4324.
Copyright (c) 2018 Georg Thieme Verlag License terms

KG Stuttgart - New York 


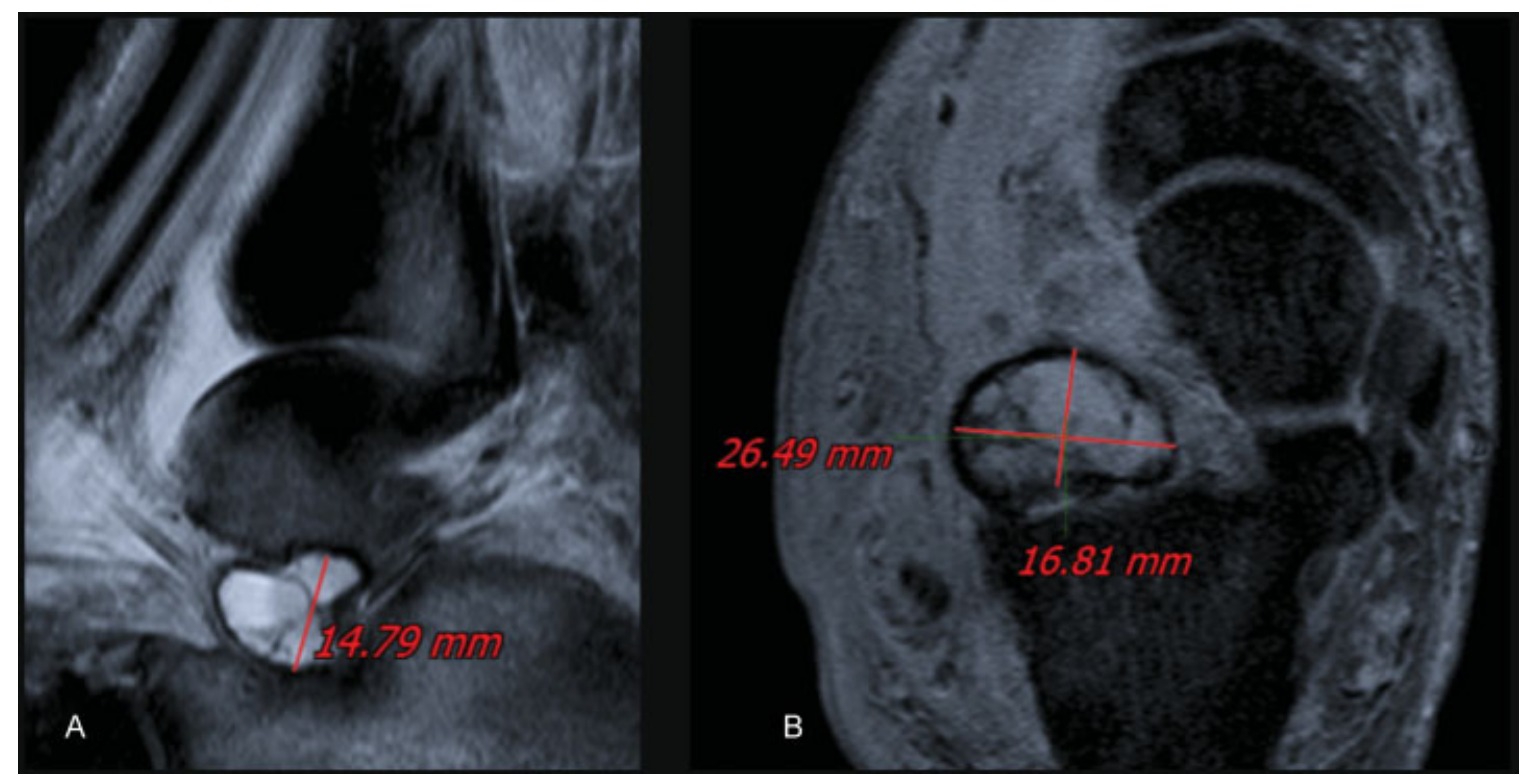

Fig. 1 Magnetic resonance imaging of the subtalar joint. Sagittal (A) and coronal (B) scans show the lesion at the tarsal canal.

a standard conservative regimen (rest, ice, compression, and elevation), due to the persistence of pain, a magnetic resonance imaging (MRI) was performed, which was evaluated as negative for pathology of the hindfoot. The patient underwent repeated periods of functional rehabilitation and physical therapy (YAG laser and tecar therapy). Two months after the trauma, there was the appearance of swelling in the anterior lateral area, in particular, at the level of the sinus tarsi, only painful during walking. In the face of this clinical evolution, corticosteroid injections were recommended.

The clinical condition progressively worsened with almost constant pain accompanied by a severe functional limitation of the ankle and hindfoot stiffness in pronation. At the clinical examination, it was evident that an anterolateral swelling at the level of sinus tarsi with a palpable tight/elastic mass was relatively fixed to the deep structures and covered by indented skin. I recommended to the patient a new MRI (at $\sim 8$ months from the previous one) which did not highlight any problem at the tarsal canal and put into evidence a mass located in the lateral portion of the tarsal canal, inhomogeneous, delimited by a pseudocapsule with respect to the surrounding tissues, without intralesional calcifications (-Fig. 1).
Based on the above-mentioned clinical and instrumental framework, open surgical removal of the mass was proposed to the patient.

\section{Surgical Procedure}

The patient was operated in semilateral decubitus. Pneumatic tourniquet was applied at the root of the thigh. A skin incision corresponding to the mass was performed and once the deep layer was reached, tissue of a lardaceous consistency and of color brownish-yellow mixed with grayishwhite was evident. A thin pseudocapsule of brown color with lamellar structure and a moderately tough texture delimited the mass (-Fig. 2A).

After the excision of the mass, there was a cavity of $\sim 3 \mathrm{~cm}$ in diameter at the lateral portion of the tarsal canal. The excised material was processed for histologic examination. In the report of the histologic examination, a giant cell osseous tumor was diagnosed (-Fig. 2B).

In the postoperative phase, a half-stitched boot was positioned with the ankle at $90^{\circ}$ and weight-bearing was not allowed for 3 weeks. Thereafter, the valve was removed and a bivalve brace was applied, and weight-bearing was
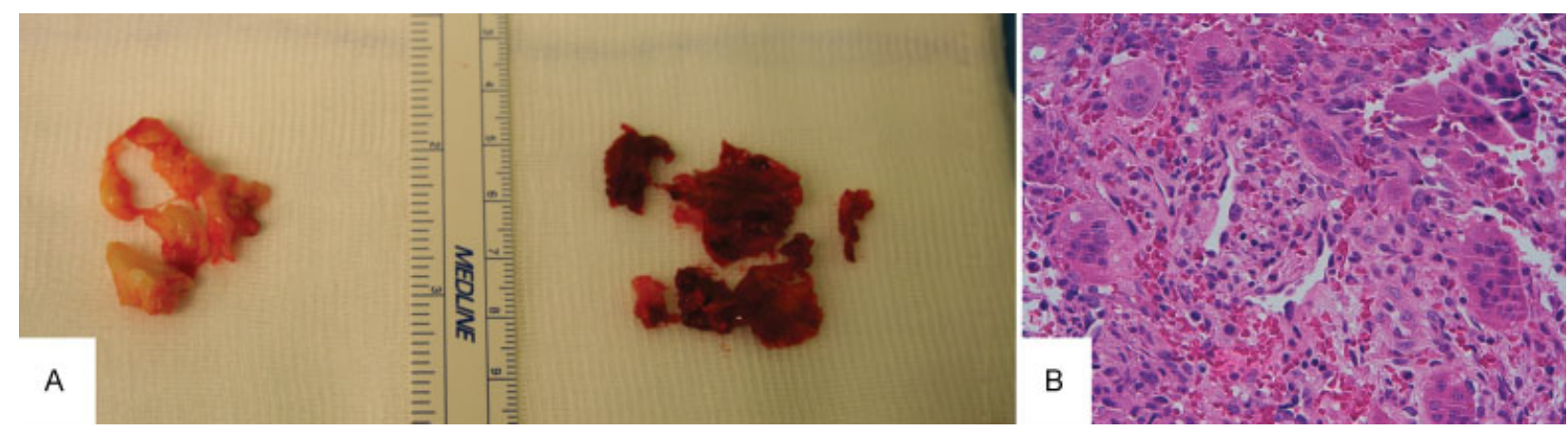

Fig. 2 Excised mass. Macroscopic appearance of the lesion (A) and histologic pattern of the pathologic tissue (B). 
allowed as tolerated. The patient performed functional rehabilitation of the ankle and subtalar joint and completed the rehabilitative program with proprioceptive exercise.

Currently, the patient no longer reports any type of pain, no range of motion restriction and no instability in both the ankle and the subtalar joint.

\section{Discussion}

The case described in the present article is rather rare and to my knowledge, no previous reports are present in the literature describing such a lesion. For this reason, only after histologic examination of the removed tissue, it was possible to speculate on the etiology of the disease.

At the origin of this type of benign tumor, there can be a "local reaction process" to a hemorrhagic problem in the tarsal canal. Probably, after an inversion ankle sprain, bleeding occurred in that district, which is richly vascularized. Such bleeding was not initially suspected in a typical clinical aspect of an ankle sprain. In a chronic phase, the development of more specific symptoms in the subtalar joint should have been evaluated with imaging studies, such as MRI. Indeed, early diagnosis would have prevented the formation of the neoformation and clinical manifestations characterized mainly by chronic pain and stiffness.

In conclusion, injuries from ankle sprain are not limited to the tibiotarsal joint, but can affect tendons, ligaments, and bones that are in close topographical correlation. When clinical course deviates from normal post-traumatic evolution, targeted instrumental investigations should be recommended. Timeliness and diagnostic accuracy improve the outcome of treatment of the lesions associated with ankle sprain.

\section{References}

1 Gerber JP, Williams GN, Scoville CR, Arciero RA, Taylor DC. Persistent disability associated with ankle sprains: a prospective examination of an athletic population. Foot Ankle Int 1998; 19(10):653-660

2 DIGiovanni BF, Fraga CJ, Cohen BE, Shereff MJ. Associated injuries found in chronic lateral ankle instability. Foot Ankle Int 2000; 21(10):809-815

3 Liu SH, Nguyen TM. Ankle sprains and other soft tissue injuries. Curr Opin Rheumatol 1999;11(02):132-137
4 Bonnel F, Toullec E, Mabit C, Tourné Y; Sofcot. Chronic ankle instability: biomechanics and pathomechanics of ligaments injury and associated lesions. Orthop Traumatol Surg Res 2010; 96(04):424-432

5 Ribbans WJ, Garde A. Tibialis posterior tendon and deltoid and spring ligament injuries in the elite athlete. Foot Ankle Clin 2013; 18(02):255-291

6 Ziai P, Benca E, Skrbensky GV, et al. The role of the medial ligaments in lateral stabilization of the ankle joint: an in vitro study. Knee Surg Sports Traumatol Arthrosc 2015;23(07):1900-1906

7 Savage-Elliott I, Murawski CD, Smyth NA, Golanó P, Kennedy JG. The deltoid ligament: an in-depth review of anatomy, function, and treatment strategies. Knee Surg Sports Traumatol Arthrosc 2013;21(06):1316-1327

8 Hintermann B, Valderrabano V, Boss A, Trouillier HH, Dick W. Medial ankle instability: an exploratory, prospective study of fifty-two cases. Am J Sports Med 2004;32(01):183-190

9 Colcuc C, Fischer S, Colcuc S, et al. Treatment strategies for partial chronic instability of the distal syndesmosis: an arthroscopic grading scale and operative staging concept. Arch Orthop Trauma Surg 2016;136(02):157-163

10 Burrus MT, Werner BC, Hadeed MM, Walker JB, Perumal V, Park JS. Predictors of peroneal pathology in Broström-Gould ankle ligament reconstruction for lateral ankle instability. Foot Ankle Int 2015;36(03):268-276

11 Harper MC. The lateral ligamentous support of the subtalar joint. Foot Ankle 1991;11(06):354-358

12 Li S-Y, Hou ZD, Zhang P, Li HL, Ding ZH, Liu YJ. Ligament structures in the tarsal sinus and canal. Foot Ankle Int 2013;34(12): $1729-1736$

13 Golanó P, Vega J, de Leeuw PA, et al. Anatomy of the ankle ligaments: a pictorial essay. Knee Surg Sports Traumatol Arthrosc 2016;24(04):944-956

14 Sarrafian SK. Biomechanics of the subtalar joint complex. Clin Orthop Relat Res 1993;(290):17-26

15 Löfvenberg R, Kärrholm J, Lundberg A. Subtalar stability in chronic lateral instability of the ankle. Foot 1992;2(01):39-43

16 Kjaersgaard-Andersen P, Wethelund JO, Helmig P, Søballe K. The stabilizing effect of the ligamentous structures in the sinus and canalis tarsi on movements in the hindfoot. An experimental study. Am J Sports Med 1988;16(05):512-516

17 Lui TH. Giant solitary synovial osteochondroma of the subtalar joint. J Foot Ankle Surg 2016;55(01):183-187

18 Dixon DJ, Monroe MT, Gabel SJ, Manoli A II. Excrescent lesion: a diagnosis of lateral talar exostosis in chronically symptomatic sprained ankles. Foot Ankle Int 1999;20(05):331-336

19 Kjaersgaard-Andersen P, Andersen K, Søballe K, Pilgaard S. Sinus tarsi syndrome: presentation of seven cases and review of the literature. J Foot Surg 1989;28(01):3-6 